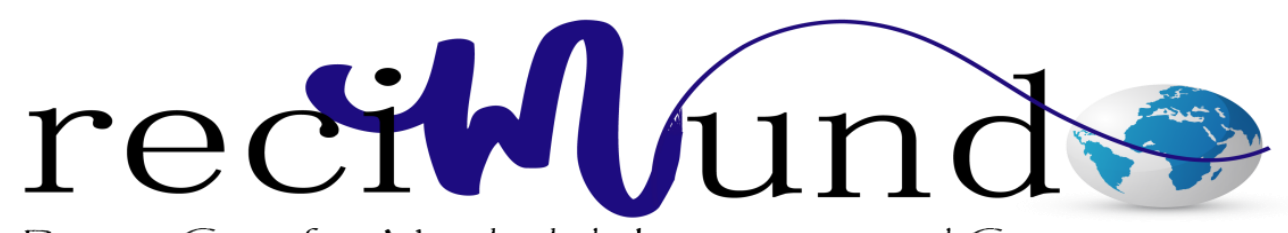

Revista Científica Mundo de la Investigación y el Conocimiento

Edison Daniel Yandun Burbano ${ }^{\text {a }}$, Luis Fernando Freire Constante ${ }^{\text {b }}$ Juan Carlos Flores $^{\text {c; }}$ Carlos Alberto Vasco Gualotuña ${ }^{\mathrm{d}}$

Responsabilidad social corporativa y su relación con la seguridad y salud ocupacional en el Ecuador

Revista Científica Mundo de la Investigación y el Conocimiento. Vol. 1 núm., 5, diciembre, 2017, pp. 766-798

DOI: $10.26820 /$ recimundo/1.5.2017.766-798

Editorial Saberes del Conocimiento 


\section{Responsabilidad social corporativa y su relación con la seguridad y salud ocupacional en el Ecuador}

Vol. 1, núm. 5., (2017)

Edison Daniel Yandun Burbano; Luis Fernando Freire Constante; Juan Carlos Flores; Carlos

Alberto Vasco Gualotuña

\section{RESUMEN}

El objetivo del presente estudio fue identificar y comparar la normativa legal ecuatoriana sobre seguridad y salud ocupacional con la Norma internacional- Guía ISO 26000 (Guía de Responsabilidad Social) en su apartado 6.4.6.2. Para verificar en qué medida la integración de la Responsabilidad Social es obligatoria en las empresas del país.

Palabras Claves: Responsabilidad social; Responsabilidad corporativa; salud ocupacional. 


\section{Responsabilidad social corporativa y su relación con la seguridad y salud ocupacional en el Ecuador}

Vol. 1, núm. 5., (2017)

Edison Daniel Yandun Burbano; Luis Fernando Freire Constante; Juan Carlos Flores; Carlos Alberto Vasco Gualotuña

\section{ABSTRACT}

The objective of this study was to identify and compare the Ecuadorian legal regulations on occupational safety and health with the International Standard - Guide ISO 26000 (Social Responsibility Guide) in section 6.4.6.2. To verify to what extent the integration of Social Responsibility is mandatory in the companies of the country.

Key Words: Social responsability; Corporate responsability; occupational health. 


\section{Responsabilidad social corporativa y su relación con la seguridad y salud ocupacional en el Ecuador}

Vol. 1, núm. 5., (2017)

Edison Daniel Yandun Burbano; Luis Fernando Freire Constante; Juan Carlos Flores; Carlos

Alberto Vasco Gualotuña

\section{Introducción.}

El termino de responsabilidad social, se caracteriza por la Globalización en el siglo XXI, en este contexto la Responsabilidad Social Empresarial (RSE) se afirma como una respuesta ante la ciudadanía por los múltiples impactos que generan las organizaciones en los ámbitos social, económico y medioambiental (Comisión Europea, 2011). Con el fin de aproximar el concepto de Responsabilidad Social y sus diversas nomenclaturas (Responsabilidad Social Empresarial, Responsabilidad Social Corporativa, Responsabilidad Social Organizacional,...) se comienza este estudio mediante la exposición de las definiciones más difundidas para, posteriormente, hacer un breve resumen desde su origen en los años 50' del siglo pasado, con las teorías más relevantes y contenidos, para desembocar al momento actual, en el que su implantación es de carácter básico y fundamental en las organizaciones debido en parte al contexto socioeconómico actual, y al desarrollo de las Tecnologías de la Información y Comunicación (TICs), con la consiguiente disposición al momento de la información y la globalización de la economía.

A día de hoy no existe una definición única consensuada a nivel internacional de la Responsabilidad Social; existen tantas definiciones como organismos involucrados en su estudio, expansión e implementación. Para contextualizar este trabajo se han contemplado las siguientes definiciones:

- "la integración voluntaria por parte de las empresas de las preocupaciones sociales y medioambientales en sus operaciones comerciales y sus relaciones con los interlocutores" (Comisión Europea, 2001)

Revista Científica Mundo de la Investigación y el Conocimiento. 1(5). pp. 766-798 


\section{Responsabilidad social corporativa y su relación con la seguridad y salud ocupacional en el Ecuador}

Vol. 1, núm. 5., (2017)

Edison Daniel Yandun Burbano; Luis Fernando Freire Constante; Juan Carlos Flores; Carlos Alberto Vasco Gualotuña

- "La responsabilidad social de la empresa (RSE) es el reflejo de la manera en que las empresas toman en consideración las repercusiones que tienen sus actividades sobre la sociedad, y en la que afirman los principios y valores por los que se rigen, tanto en sus propios métodos y procesos internos como en su relación con los demás actores. La RSE es una iniciativa de carácter voluntario y que sólo depende de la empresa, y se refiere a actividades que se considera rebasan el mero cumplimiento de la legislación." (Organizacíón Internacional del Trabajo, 2006)

- "La responsabilidad de una organización ante los impactos que sus decisiones y actividades ocasionan en la sociedad y el medio ambiente, mediante un comportamiento ético y transparente que: 1) contribuya al desarrollo sostenible incluyendo la salud y el bienestar de la sociedad; 2) tome en consideración las expectativas de sus partes interesadas; 3) cumpla con la legislación aplicable y sea coherente con la normativa internacional de comportamiento; y 4) esté integrada en toda la organización y se lleve a la práctica en sus relaciones" (ISO 26000, 2010).

Los lineamientos definidos en los objetivos 1, 2, 3, 4, 6 y 11 del Plan Nacional del Buen Vivir en el Ecuador (PNBV) promulgado por el gobierno, promueve el desarrollo de una política pública que fortalezca el talento humano como una medida sistémica que conduzca a la transformación en el desarrollo nacional. Esta transformación se basa en el cambio de la matriz productiva, que según la Secretaría Nacional de Planificación y Desarrollo (SENPLADES) consiste en pasar de una economía con especialización primaria, a una economía que privilegie 


\section{Responsabilidad social corporativa y su relación con la seguridad y salud ocupacional en el Ecuador}

Vol. 1, núm. 5., (2017)

Edison Daniel Yandun Burbano; Luis Fernando Freire Constante; Juan Carlos Flores; Carlos

Alberto Vasco Gualotuña

$\overline{\text { la producción con valor agregado y que se haya diversificado con base en modelos de nuevos }}$ conocimientos.

Un aspecto importante a considerar en este proceso propuesto es que toda actividad empresarial depende directamente del capital humano por lo tanto es prioridad para las empresas el cuidarlo en referencia a garantizar las condiciones mínimas de seguridad y salud para llevar a cabo actividades operativas, una guía para respetar estos principios básicos es la legislación que compete a la seguridad y salud ocupacional en Ecuador, entre estas se puede mencionar puede mencionar:

El artículo 326 numeral 5 de la Constitución de la República, establece que: "Toda persona tendrá derecho a desarrollar sus labores en un ambiente adecuado y propicio, que garantice su salud, integridad, seguridad, higiene y bienestar"; (Nacional, 2008) y, el numeral 6 establece que: "Toda persona rehabilitada después de un accidente de trabajo o enfermedad, tendrá derecho a ser reintegrada al trabajo y a mantener la relación laboral, de acuerdo con la ley". (Nacional, 2008)

El Reglamento de Seguridad y Salud de los Trabajadores y Mejoramiento del Ambiente de Trabajo manifiesta en su artículo 15 que "En las empresas permanentes que cuenten con cien o más trabajadores estables, se deberá contar con una Unidad de Seguridad e Higiene, dirigida por un técnico en la materia que reportará a la más alta autoridad de la empresa o entidad. En las empresas o Centros de Trabajo calificados de alto riesgo por el Comité Interinstitucional, que tengan un número inferior a cien trabajadores, pero mayor de cincuenta, se deberá contar con un 


\section{Responsabilidad social corporativa y su relación con la seguridad y salud ocupacional en el Ecuador}

Vol. 1, núm. 5., (2017)

Edison Daniel Yandun Burbano; Luis Fernando Freire Constante; Juan Carlos Flores; Carlos Alberto Vasco Gualotuña

técnico en seguridad e higiene del trabajo. De acuerdo al grado de peligrosidad de la empresa, el Comité podrá exigir la conformación de un Departamento de Seguridad e Higiene." (Decreto Ejecutivo, 1986)

El Ecuador es miembro de la Comunidad Andina; y, la Decisión 584 del Consejo Andino de Ministros de Relaciones Exteriores; y, la Resolución 957 de la Secretaría General de la Comunidad Andina, señalan para los países que integran la Comunidad Andina normas fundamentales en materia de seguridad y salud en el trabajo, que tienen como objeto promover y regular acciones a desarrollarse para disminuir o eliminar los daños a la salud del trabajador mediante aplicación de medidas de control, y el desarrollo de las actividades necesarias para la prevención de riesgos derivados del trabajo;

El Código de Trabajo señala la normativa para verificar el cumplimiento técnico y legal en materia de seguridad y salud en el trabajo en el Capítulo V del Título IV, según lo señalado en los artículos 412, 434, 435, 436; y lo establecido en el artículo 42 en los numerales 2 y3.

En el Reglamento del Seguro General de Riesgos del Trabajo Resolución CD 513 se hablas de condiciones mínimas y principios de acción preventiva que debe aplicar una organización y puede acompañarse de normas emitidas por el Instituto Ecuatoriano de Normalización (INEN) que brindan lineamientos en varios aspectos a la Seguridad y Salud en el Trabajo.

Estos cuerpos legales han ayudado a:

Revista Científica Mundo de la Investigación y el Conocimiento. 1(5). pp. 766-798 


\section{Responsabilidad social corporativa y su relación con la seguridad y salud ocupacional en el Ecuador}

Vol. 1, núm. 5., (2017)

Edison Daniel Yandun Burbano; Luis Fernando Freire Constante; Juan Carlos Flores; Carlos

Alberto Vasco Gualotuña

- Promover la prevención en las organizaciones desde un punto de vista técnico y legal con su respectiva integración en la misma.

- Realizar la identificación de peligros, evaluaciones y análisis de riesgos proponiendo medidas preventivas para poder eliminar o minimizar el impacto que puedan tener los colaboradores, la infraestructura y el entorno de las organizaciones.

- Planificar actividades de información y formación de trabajadores en prevención de riesgos.

- Verificar el cumplimiento del programa de control y reducción de riesgos.

- Dirigir las actuaciones a desarrollar en casos de emergencia y primeros auxilios.

Lo que se pretende con estos lineamientos que son de carácter legar es aplicar los programas preventivos que garanticen la seguridad y salud y que la organización busque cumplir con un parámetro básico de responsabilidad que se traduce en garantizar la vida al trabajador.

En la Tabla 1 se encuentra la nomenclatura de la legislación aplicable en el Ecuador, estos cuerpos legales son parte de la legislación de seguridad y salud y son en su mayoría de carácter general, no se concentra en un sector en especial debido a que en este estudio se busca abarcar a todo tipo de organización. 


\section{Responsabilidad social corporativa y su relación con la seguridad y salud ocupacional en el Ecuador}

Vol. 1, núm. 5., (2017)

Edison Daniel Yandun Burbano; Luis Fernando Freire Constante; Juan Carlos Flores; Carlos Alberto Vasco Gualotuña

\begin{tabular}{|c|l|}
\hline \multicolumn{2}{|c|}{ Legislación: nomenclaturas } \\
\hline C.R.E. & Constitución de la República del Ecuador \\
\hline D-584 & $\begin{array}{l}\text { Decisión 584. } \\
\text { Instrumento Andino de Seguridad y Salud en el Trabajo }\end{array}$ \\
\hline R-957 & $\begin{array}{l}\text { Resolución 957. } \\
\text { Reglamento del Instrumento Andino de Seguridad y Salud en el Trabajo }\end{array}$ \\
\hline C.T. & Código del Trabajo \\
\hline D.E.-2393 & $\begin{array}{l}\text { Decreto Ejecutivo 2393. } \\
\text { Reglamento de Seguridad y Salud de los Trabajadores y Mejoramiento } \\
\text { del Medio Ambiente de Trabajo }\end{array}$ \\
\hline C. D. 513 & $\begin{array}{l}\text { Resolución N }{ }^{\circ} \text { C. D. 513. } \\
\text { Reglamento Seguro General de Riesgos del Trabajo }\end{array}$ \\
\hline A-1404 & $\begin{array}{l}\text { Reglamento para el Funcionamiento de los Servicios Médicos de } \\
\text { Empresas }\end{array}$ \\
\hline A.M.- 398 & Acuerdo Ministerial 398 VIH-Sida - Ministerio Del Trabajo \\
\hline
\end{tabular}

\section{Tabla 1 Nomenclatura de legislación aplicable en el Ecuador}

\section{Metodología.}

Estudio comparativo de tipo observacional a partir de fuentes de información secundaria; normativa legal ecuatoriana vigente aplicable sobre Seguridad y Salud Ocupacional y la Norma internacional ISO 26000 (Guía de Responsabilidad Social) concretado en su apartado 6.4.6.2.

Se procedió a la identificación y análisis de la normativa legal (noviembre 2016) en esta materia, para su posterior comparación en cada una de las recomendaciones incluidas en el apartado indicado de la Norma ISO 26000. 


\section{Responsabilidad social corporativa y su relación con la seguridad y salud ocupacional en el Ecuador}

Vol. 1, núm. 5., (2017)

Edison Daniel Yandun Burbano; Luis Fernando Freire Constante; Juan Carlos Flores; Carlos

Alberto Vasco Gualotuña

Con el resultado del análisis de la normativa legal se procedido a elaborar en cuestionario en el cual se levantaron 22 preguntas y este se lo aplico luego de determinar el tamaño de la muestra considerando el número total de empresas resultando para el presente estudio 384 muestras, basada sobre un universo de 842.936 empresas a nivel nacional, con un intervalo de confianza del 95\% y un margen de error del 5\%. Con los datos obtenidos se realiza el análisis de los resultados en los distintos tipos de empresas y se procede a la elaboración del documento.

\begin{tabular}{|c|l|l|}
\hline $\mathbf{N}^{\circ}$ & \multicolumn{1}{|c|}{ FASES DE ESTUDIO } & \multicolumn{1}{c|}{ DESCRIPCIÓN } \\
\hline 1 & Revisión bibliográfica. & $\begin{array}{l}\text { Revisión de bibliográfica de la temática del caso } \\
\text { de estudio. } \\
\text { Revisión del marco legal. } \\
\text { Revisión de metodologías }\end{array}$ \\
\hline 2 & $\begin{array}{l}\text { Identificación y análisis de la } \\
\text { normativa legal (noviembre 2016) }\end{array}$ & $\begin{array}{l}\text { Se procedió a la identificación y análisis de la } \\
\text { normativa legal (noviembre 2016) en esta } \\
\text { materia, para su posterior comparación en cada } \\
\text { una de las recomendaciones incluidas en el } \\
\text { apartado indicado de la Norma ISO 26000. }\end{array}$ \\
\hline 3 & Diseño y creación de Cuestionario & $\begin{array}{l}\text { Elaboración de un cuestionario en el cual se } \\
\text { levantaron 22 preguntas. }\end{array}$ \\
\hline 4 & Aplicación de cuestionario & $\begin{array}{l}\text { Aplicación del cuestionario a una muestra de } \\
384 \text { empresas basada sobre un universo de } \\
842.936 \text { empresas a nivel nacional, con un } \\
\text { intervalo de confianza del 95\% y un margen de } \\
\text { error del 5\%. }\end{array}$ \\
\hline 5 & Análisis de resultados & $\begin{array}{l}\text { Con los datos obtenidos se realiza el análisis de } \\
\text { los resultados en los distintos tipos de empresas } \\
\text { yse procede a la elaboración del documento. }\end{array}$ \\
\hline 6 & Presentación del documento. & \\
\hline
\end{tabular}

Tabla 2 Fases del estudio 


\section{Responsabilidad social corporativa y su relación con la seguridad y salud ocupacional en el Ecuador}

Vol. 1, núm. 5., (2017)

Edison Daniel Yandun Burbano; Luis Fernando Freire Constante; Juan Carlos Flores; Carlos Alberto Vasco Gualotuña

\section{Técnicas para recoger la información}

El presente proyecto utilizará el método deductivo que va de lo general a lo particular, partiendo de datos generales aceptados como valederos para deducir por medio del razonamiento lógico varias suposiciones es decir; parte de verdades previamente establecidas como principios generales para luego aplicarlos a casos individuales y comprobar así su validez. Las técnicas o herramientas para la recolección de información pueden ser empleadas de manera integrada. Las técnicas que se manejan en el presente proyecto son:

- Entrevista.- Una técnica orientada a establecer contacto directo con las personas que se consideren fuente de información, si bien puede soportarse en un cuestionario muy flexible, tiene como propósito obtener información más espontánea y abierta. Durante la misma, puede profundizarse la información de interés para el estudio.

- $\quad$ Encuesta. - Es una de las técnicas de recolección de información más usadas, a pesar de que cada vez pierde mayor credibilidad por el sesgo de las personas encuestadas. La encuesta se fundamenta en un cuestionario o conjunto de preguntas que se preparan con el propósito de obtener información de las personas.

- $\quad$ Observación directa.- La observación directa cobra mayor credibilidad y su uso tiende a generalizarse, debido a que permite obtener información real y confiable. 


\section{Responsabilidad social corporativa y su relación con la seguridad y salud ocupacional en el Ecuador}

Vol. 1, núm. 5., (2017)

Edison Daniel Yandun Burbano; Luis Fernando Freire Constante; Juan Carlos Flores; Carlos

Alberto Vasco Gualotuña

Análisis de documentos.- Técnica basada en fichas bibliográficas que tienen como propósito analizar material impreso, la separación de las partes de un todo, a fin de ser estudiadas por separado.

Novedad Científica de los resultados

El presente proyecto de tesis doctoral evalúa de manera cualitativa y cuantitativa el alcance de la legislación ecuatoriana en materia de Seguridad y Salud Ocupacional analizando y comprobando si los contenidos exigibles en la ISO 26000 están reflejados en la normativa que aplica en el Ecuador, no existe un estudio similar en la actualidad debido a que los temas de seguridad y salud y responsabilidad social corporativa son relativamente nuevos es así que el INEC Instituto Nacional de Estadística y Censos del Ecuador desde el año 1990 ha sido el organismo encargado de generar información estadística, ha aplicado encuestas y realizado investigaciones en diferentes ámbitos sociodemográficos, económicos, sociales y ambientales del país permitiendo diagnosticar la realidad socioeconómica, de empleo y ambiente en el país pero no existe ningún estudio que este en relación directa con las seguridad y salud ocupacional y la responsabilidad social corporativa.

\section{Revisión.}

Dentro de la matriz productiva vigente en el Ecuador se han identificado 14 sectores productivos y 5 industrias estratégicas para el proceso de cambio de dicha matriz. Para el efecto se ha considerado que los sectores priorizados y las industrias estratégicas serán los que faciliten la articulación efectiva de la política pública y la materialización de esta transformación. Los 


\section{Responsabilidad social corporativa y su relación con la seguridad y salud ocupacional en el Ecuador}

Vol. 1, núm. 5., (2017)

Edison Daniel Yandun Burbano; Luis Fernando Freire Constante; Juan Carlos Flores; Carlos Alberto Vasco Gualotuña

requerimientos legales de seguridad y salud deben contribuir en la gestión de los ambientes laborales para garantizar que estos sean sanos y saludables, y desde el punto de vista de la investigación por medio del conocimiento del impacto que genera el ambiente laboral en el trabajador podrá diseñar e implementar sistemas de gestión que garanticen la salud y seguridad de los trabajadores en cualquier actividad económica y por ende al cumplir con estas actividades que son parte de la empresa responsable.

La seguridad y salud se articulan con las siguientes áreas en algunas de las ramas económicas, así como en la protección contra riesgos específicos.

Industrias estratégicas (consideradas dentro de la salud y seguridad ocupacional como industrias de alto riesgo).

$$
\begin{array}{ll}
- & \text { Astillero } \\
- & \text { Refinería } \\
- & \text { Petroquímica } \\
- & \text { Siderurgia } \\
- & \text { Metalurgia }
\end{array}
$$

Áreas de interés público

\section{- Salud}

- Ciencias fundamentales

- $\quad$ Seguridad ciudadana

- $\quad$ Otras áreas de coyuntura de acuerdo a políticas públicas

Subsectores priorizados de la producción 


\section{Responsabilidad social corporativa y su relación con la seguridad y salud ocupacional en el Ecuador}

Vol. 1, núm. 5., (2017)

Edison Daniel Yandun Burbano; Luis Fernando Freire Constante; Juan Carlos Flores; Carlos

Alberto Vasco Gualotuña

\section{- Turismo}

- $\quad$ Alimentos frescos y procesados

- $\quad$ Energías Renovables (Bioenergía y Alternativas)

- $\quad$ Biotecnología (Bioquímica y Biomedicina)

- $\quad$ Servicios ambientales

- $\quad$ Metalmecánica

- Tecnología (Hardware y Software)

- Plásticos de caucho sintético

- $\quad$ Confecciones y calzado

- Vehículos, automotores, autopartes

- $\quad$ Transporte y logística

- Construcción

- $\quad$ Cadena agroforestal sustentable y productos elaborados

- $\quad$ Productos farmacéuticos y químicos.

Según la Encuesta Nacional de Empleo, Desempleo y Subempleo ENEMDU ${ }^{1}$ de marzo 2016 por población económicamente activa (PEA) son consideradas aquellas "personas de 15 años y más que trabajaron al menos 1 hora en la semana de referencia o, aunque no trabajaron, tuvieron trabajo (empleados); y personas que no tenían empleo pero estaban disponibles para trabajar y buscan empleo (desempleados)".

\footnotetext{
1 http://www.ecuadorencifras.gob.ec/empleo-encuesta-nacional-de-empleo-desempleo-ysubempleo-enemdu/
}

Revista Científica Mundo de la Investigación y el Conocimiento. 1(5). pp. 766-798 


\section{Responsabilidad social corporativa y su relación con la seguridad y salud ocupacional en el Ecuador}

Vol. 1, núm. 5., (2017)

Edison Daniel Yandun Burbano; Luis Fernando Freire Constante; Juan Carlos Flores; Carlos Alberto Vasco Gualotuña

\section{Gráfico 1 Población económicamente activa (PEA) 2016}

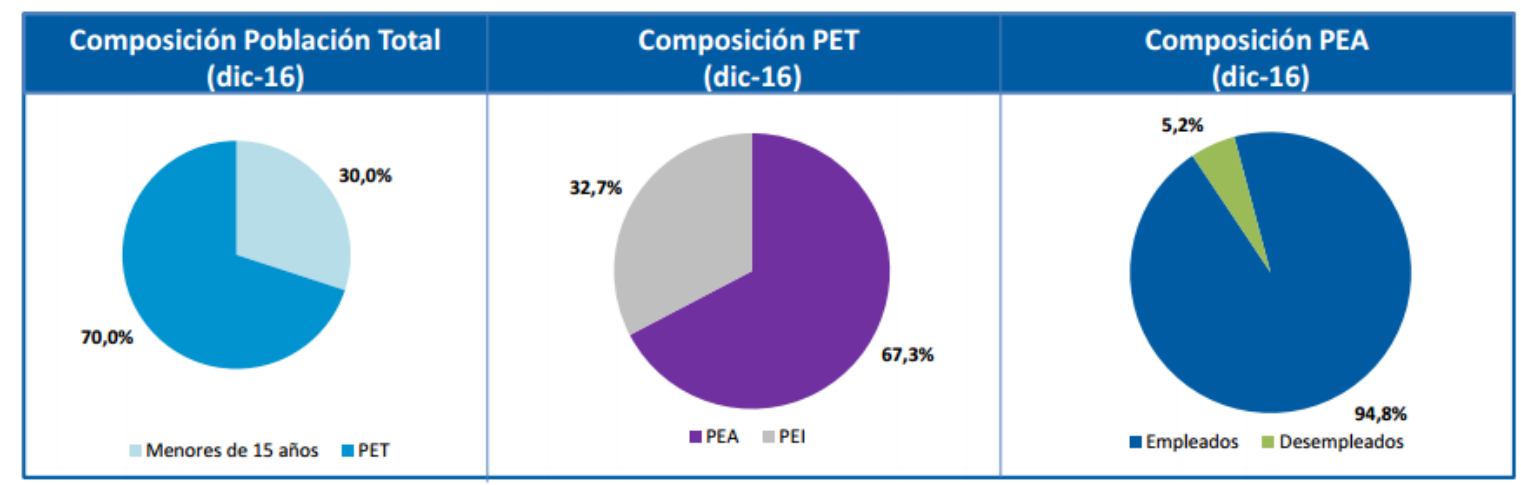

PET= Población en edad de trabajar, PEA= Población económicamente activa, PEI= Población económicamente inactiva.

*La categoría de empleo incluye a los asalariados e independientes

Fuente: Encuesta Nacional de Empleo, Desempleo y Subempleo (ENEMDU) 2016.

Con este antecedente, se puede apreciar la siguiente información:

De la población total, el 70,0\% está en edad de trabajar.

El 67,3\% de la población en edad de trabajar se encuentra económicamente activa.

De la población económicamente activa, el 94,8\% son personas con empleo.

Por lo tanto y de acuerdo a la información presentada:

Revista Científica Mundo de la Investigación y el Conocimiento. 1(5). pp. 766-798 


\section{Responsabilidad social corporativa y su relación con la seguridad y salud ocupacional en el Ecuador}

Vol. 1, núm. 5., (2017)

Edison Daniel Yandun Burbano; Luis Fernando Freire Constante; Juan Carlos Flores; Carlos

Alberto Vasco Gualotuña

La población en edad de trabajar (PET) es de 11,7 millones de personas.

La población económicamente activa (PEA) es de 7,9 millones de personas.

La población económicamente inactiva (PEI) es de 3,8 millones de personas.

Con la información descrita anteriormente y haciendo referencia al número de empresas registradas a diciembre del 2014 es de 843644 cuya clasificación en el siguiente gráfico nos reflejan los siguientes datos:

\section{Gráfico 2 Estructura de empresas según su tamaño}

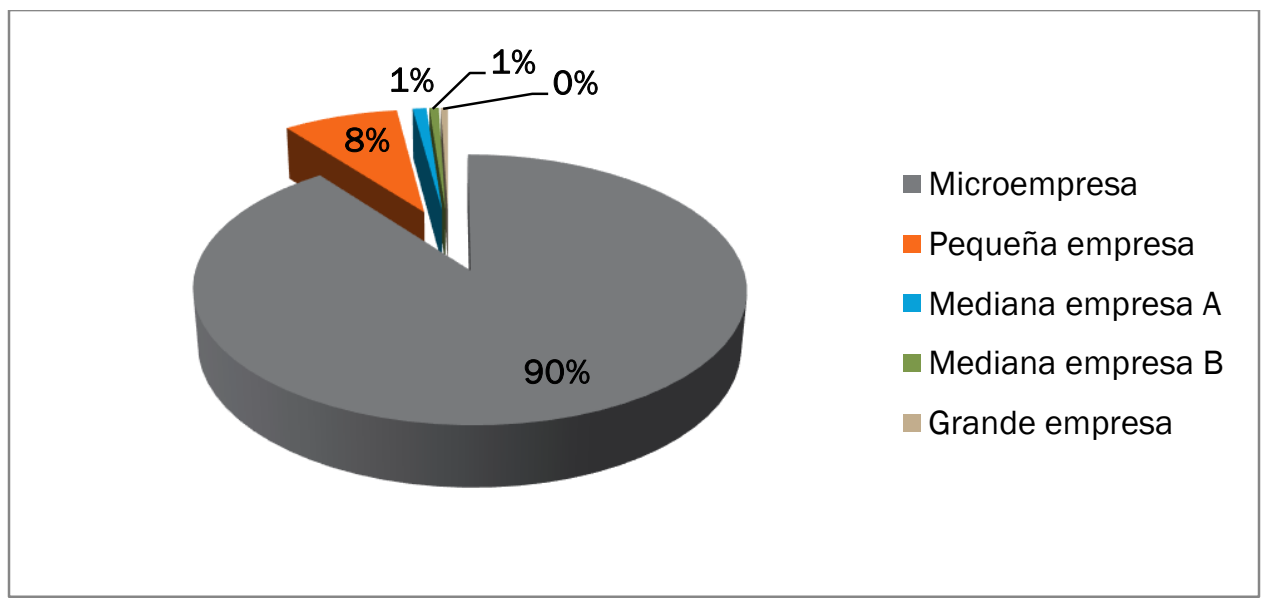

Tabla 1 Estructura de empresas según su tamaño

\begin{tabular}{|l|l|l|l|l|}
\hline- & $\mathbf{2 0 1 2}$ & $\mathbf{2 0 1 3}$ & $\mathbf{2 0 1 4}$ & $\mathbf{2 0 1 5}$ \\
\hline Microempresa & 656.406 & 731.073 & 760.472 & 764.001 \\
\hline Pequeña empresa & 59.399 & 61.785 & 65.330 & 61.987 \\
\hline Mediana empresa A & 7.102 & 7.583 & 7.961 & 7.733 \\
\hline Mediana empresa B & 4.862 & 5.244 & 5.632 & 5.156 \\
\hline
\end{tabular}




\section{Responsabilidad social corporativa y su relación con la seguridad y salud ocupacional en el Ecuador}

Vol. 1, núm. 5., (2017)

Edison Daniel Yandun Burbano; Luis Fernando Freire Constante; Juan Carlos Flores; Carlos Alberto Vasco Gualotuña

\begin{tabular}{|l|l|l|l|l|}
\hline Grande empresa & 3.591 & 3.886 & 4.228 & 4.059 \\
\hline TOTAL & 731.360 & 809.571 & 843.623 & 842.936
\end{tabular}

Fuente: Instituto Nacional de Estadísticas y Censos (INEC). (2016). Disponible en:

http://www.ecuadorencifras.gob.ec/directoriodeempresas/.

Si realizamos un diagnóstico de las condiciones de salud y trabajo de la población económicamente activa en Ecuador, con relación a los diferentes niveles de desarrollo de los países del mundo, ello reflejaría el aumento en la conciencia que se tiene acerca de la importancia de la optimización de la salud del trabajador y las condiciones de trabajo para el desarrollo progresivo del aparato productivo. Años atrás la seguridad y salud de los trabajadores se la manejaba de manera intuitiva, sin bases técnicas y científicas, dirigida por personal sin capacitación específica en esta área. Esta situación provocó altos índices de siniestralidad laboral como accidentes de trabajo y enfermedades profesionales.

Si bien es cierto el país cuenta con el Instituto Ecuatoriano de Seguridad Social IESS, como medio de protección de los empleados bajo relación de dependencia, ya sea trabajando en las entidades públicas y del sector privado, tal como se muestra en el gráfico siguiente:

Gráfico 3 Estructura de empresas según su tamaño 


\section{Responsabilidad social corporativa y su relación con la seguridad y salud ocupacional en el Ecuador}

Vol. 1, núm. 5., (2017)

Edison Daniel Yandun Burbano; Luis Fernando Freire Constante; Juan Carlos Flores; Carlos

Alberto Vasco Gualotuña

Durante diciembre 2016, el 32,5\% de Empleados se encuentran afiliados al IESS- Seguro

General, mientras que el $\mathbf{5 5 , 9 \%}$ no tiene ninguna afiliación.

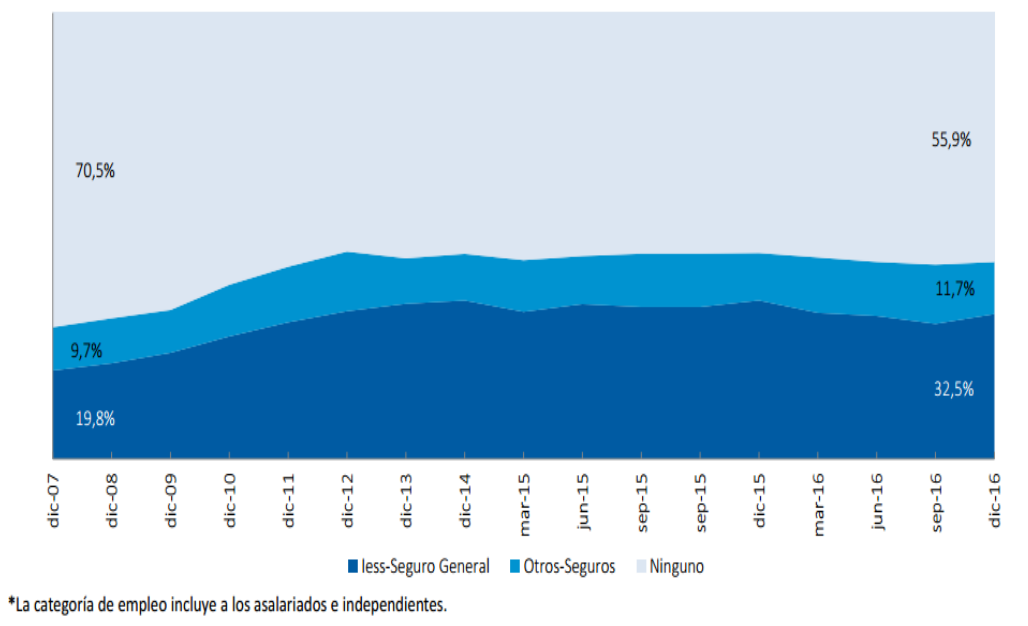

Con la información disponible, podemos decir que únicamente de ese $32,5 \%$ de empleados afiliados al IESS, se cuenta con estadísticas parciales referentes a los indicadores de siniestralidad.

En el Ecuador no se tiene un registro de los accidentes industriales, únicamente se tiene un registro de accidentes y enfermedades laborales (IESS)2, los cuales son solo una parte de lo que representan a los eventos que generan pérdidas a la sociedad ecuatoriana.

Los accidentes de trabajo (A.T.) y enfermedades profesionales (E. P.) tienen un crecimiento casi exponencial y específicamente a partir del 2010 se observa un mayor crecimiento. Esto se debe a los sistemas de control y notificación establecidos por el Seguro General de Riesgos del Trabajo del IESS al generar nuevos marcos legales que obligan a las

2 IESS Instituto Ecuatoriano de Seguridad Social

Revista Científica Mundo de la Investigación y el Conocimiento. 1(5). pp. 766-798 


\section{Responsabilidad social corporativa y su relación con la seguridad y salud ocupacional en el Ecuador}

Vol. 1, núm. 5., (2017)

Edison Daniel Yandun Burbano; Luis Fernando Freire Constante; Juan Carlos Flores; Carlos Alberto Vasco Gualotuña

empresas a evidenciar su gestión y sobre todo se realza la importancia de la obligación de los empleadores a reportar los A.T. y las presunciones de E.P.

Gráfico 4 distribución de A.T. y E.P. 2006-2014

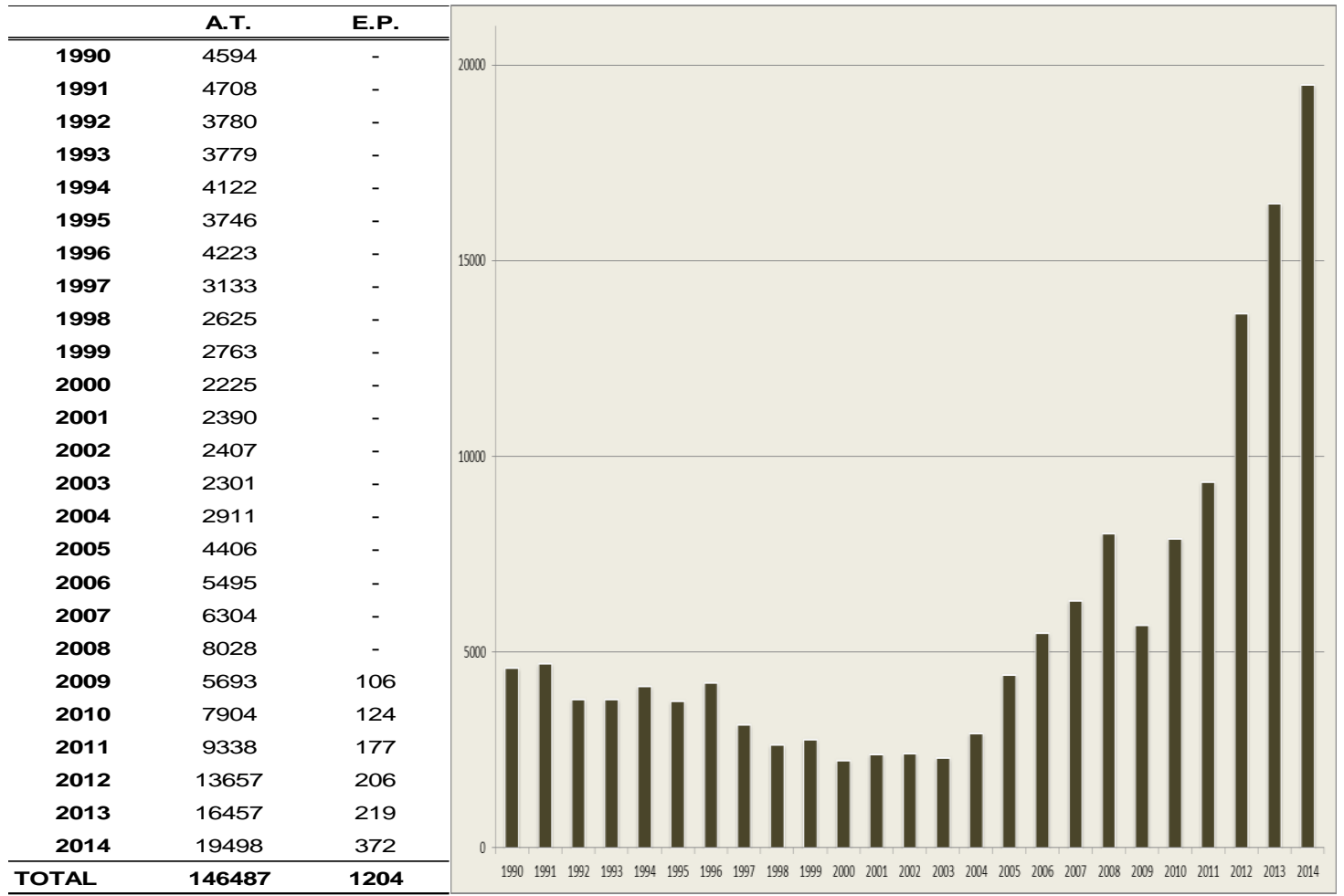

Fuente: IESS Instituto Ecuatoriano de Seguridad Social

El gestionar estas cifras requiere de profesionales con un sólido conocimiento profesional de gestión e investigación para minimizar el impacto de las consecuencias de accidentes en las 


\section{Responsabilidad social corporativa y su relación con la seguridad y salud ocupacional en el Ecuador}

Vol. 1, núm. 5., (2017)

Edison Daniel Yandun Burbano; Luis Fernando Freire Constante; Juan Carlos Flores; Carlos

Alberto Vasco Gualotuña

empresas o industrias. De todas maneras esta información demuestra la necesidad de una gestión técnica en la prevención de los riesgos, sistemas gestión enfocados a la seguridad industrial, y lo más importante que las empresas sean responsables en sus actividades laborales frente a sus colaboradores.

Con la creación de estrictas leyes que protegen al empleado y mayor exigencia de cuidado por parte del empleador, la presión social y la demanda de los empleados por puestos de trabajos más seguros, la necesidad de aplicar prácticas de seguridad y salud ocupacional se hizo inminente es por ello que la preocupación cada vez mayor por precautelar la seguridad de los trabajadores que acoge todos los ámbitos en los que puede ser protegido en sus horas de labor.

A continuación se muestran las tablas con la información generada por el Instituto Ecuatoriano de Seguridad Social (IESS) en donde se expone cómo han evolucionado los accidentes de trabajo y las enfermedades ocupacionales de la población afiliada en el Ecuador entre los años 2013-2015.

Tabla 2 tasa de incidencia (TI) de accidentes de trabajo (at) y enfermedades profesionales (E.P.) según provincia, año 2013.

\begin{tabular}{|c|c|c|c|c|c|c|c|c|c|c|}
\hline \multirow{3}{*}{ Provincia } & \multicolumn{2}{|c|}{ A.T. } & \multicolumn{2}{|c|}{ A.T. } & \multirow{2}{*}{$\begin{array}{l}\text { T.I. } \\
\text { A.T }\end{array}$} & \multicolumn{2}{|c|}{ E.P. } & \multicolumn{2}{|c|}{ E.P. } & \multirow{2}{*}{$\begin{array}{l}\text { T.I. } \\
\text { E.P }\end{array}$} \\
\hline & \multicolumn{2}{|c|}{ Notificados } & \multicolumn{2}{|c|}{ Calificados } & & \multicolumn{2}{|c|}{ Notificados } & \multicolumn{2}{|c|}{ Calificados } & \\
\hline & $\mathrm{n}$ & $\%$ & $\mathrm{n}$ & $\%$ & & $\mathrm{n}$ & $\%$ & $\mathrm{n}$ & $\%$ & \\
\hline Azuay & 709 & $3 \%$ & 69 & $2 \%$ & 45,7 & 11 & $2 \%$ & 0 & $0 \%$ & 0,0 \\
\hline Bolívar & 114 & $1 \%$ & 5 & $0 \%$ & 33,5 & 0 & $0 \%$ & 0 & $0 \%$ & 0,0 \\
\hline Cañar & 480 & $2 \%$ & 0 & $0 \%$ & 0,0 & 2 & $0 \%$ & 0 & $0 \%$ & 0,0 \\
\hline Carchi & 51 & $0 \%$ & 0 & $0 \%$ & 0,0 & 1 & $0 \%$ & 0 & $0 \%$ & 0,0 \\
\hline Chimborazo & 168 & $1 \%$ & 7 & $0 \%$ & 15,3 & 0 & $0 \%$ & 0 & $0 \%$ & 0,0 \\
\hline
\end{tabular}

Revista Científica Mundo de la Investigación y el Conocimiento. 1(5).pp. 766-798 


\section{Responsabilidad social corporativa y su relación con la seguridad y salud ocupacional en el Ecuador}

Vol. 1, núm. 5., (2017)

Edison Daniel Yandun Burbano; Luis Fernando Freire Constante; Juan Carlos Flores; Carlos Alberto Vasco Gualotuña

\begin{tabular}{lllllllllll}
\hline Cotopaxi & 301 & $1 \%$ & 1 & $0 \%$ & 2,0 & 48 & $8 \%$ & 0 & $0 \%$ & 0,0 \\
El Oro & 377 & $2 \%$ & 3 & $0 \%$ & 3,5 & 5 & $1 \%$ & 0 & $0 \%$ & 0,0 \\
Esmeraldas & 473 & $2 \%$ & 13 & $0 \%$ & 29,4 & 3 & $0 \%$ & 0 & $0 \%$ & 0,0 \\
Galápagos & 34 & $0 \%$ & 0 & $0 \%$ & 0,0 & 7 & $1 \%$ & 0 & $0 \%$ & 0,0 \\
Guayas & 9.758 & $47 \%$ & 133 & $3 \%$ & 19,2 & 72 & $11 \%$ & 0 & $0 \%$ & 0,0 \\
Imbabura & 129 & $1 \%$ & 0 & $0 \%$ & 0,0 & 13 & $2 \%$ & 0 & $0 \%$ & 0,0 \\
Loja & 222 & $1 \%$ & 0 & $0 \%$ & 0,0 & 23 & $4 \%$ & 0 & $0 \%$ & 0,0 \\
Los Ríos & 952 & $5 \%$ & 1 & $0 \%$ & 1,6 & 3 & $0 \%$ & 0 & $0 \%$ & 0,0 \\
Manabí & 441 & $2 \%$ & 3 & $0 \%$ & 2,1 & 2 & $0 \%$ & 0 & $0 \%$ & 0,0 \\
Morona Santiago & 67 & $0 \%$ & 0 & $0 \%$ & 0,0 & 0 & $0 \%$ & 0 & $0 \%$ & 0,0 \\
Napo & 441 & $2 \%$ & 15 & $0 \%$ & 125,0 & 19 & $3 \%$ & 0 & $0 \%$ & 0,0 \\
Orellana & 213 & $1 \%$ & 0 & $0 \%$ & 0,0 & 32 & $5 \%$ & 0 & $0 \%$ & 0,0 \\
Pastaza & 80 & $0 \%$ & 0 & $0 \%$ & 0,0 & 0 & $0 \%$ & 0 & $0 \%$ & 0,0 \\
Pichincha & 4.671 & $22 \%$ & 4.080 & $94 \%$ & 431,5 & 356 & $57 \%$ & 133 & $100 \%$ & 14,1 \\
Santa Elena & 165 & $1 \%$ & 0 & $0 \%$ & 0,0 & 2 & $0 \%$ & 0 & $0 \%$ & 0,0 \\
Santo & & & & & 0 & & $0 \%$ \\
Tsáchilas & 291 & $1 \%$ & 0 & $0 \%$ & 0,0 & 2 & $0 \%$ & 0 & $0 \%$ & 0,0 \\
Sucumbíos & 216 & $1 \%$ & 22 & $1 \%$ & 101,6 & 10 & $2 \%$ & 0 & $0 \%$ & 0,0 \\
Tungurahua & 307 & $1 \%$ & 10 & $0 \%$ & 12,2 & 14 & $2 \%$ & 0 & $0 \%$ & 0,0 \\
Zamora Chinchipe & 125 & $1 \%$ & 1 & $0 \%$ & 8,2 & 5 & $1 \%$ & 0 & $0 \%$ & 0,0 \\
\hline & 20.785 & $100 \%$ & 4.363 & $100 \%$ & 165,3 & 630 & $100 \%$ & 133 & $100 \%$ & 5,0 \\
\hline
\end{tabular}

Fuente: Datos obtenidos a través de la página web del Instituto Ecuatoriano de Seguridad Social (IESS) - Seguro General de Riesgos del Trabajo (SGRT) - Estadísticas del Seguro de Riesgos del Trabajo para el período 2013 a 2015.

http://sart.iess.gob.ec/SRGP/indicadores_ecuador.php Consulta: 21 de abril de 2016

Tabla 3Ttasa de incidencia (TI) de accidentes de trabajo (AT) y enfermedades profesionales (E.P.) según provincia, año 2014.

\begin{tabular}{|c|c|c|c|c|c|c|c|c|c|c|}
\hline \multirow[t]{2}{*}{ Provincia } & \multicolumn{2}{|c|}{$\begin{array}{c}\text { A.T. } \\
\text { Notificados }\end{array}$} & \multicolumn{2}{|c|}{$\begin{array}{c}\text { A.T. } \\
\text { Calificados }\end{array}$} & \multirow[t]{2}{*}{$\begin{array}{l}\text { T.I. } \\
\text { A.T. }\end{array}$} & \multicolumn{2}{|c|}{$\begin{array}{c}\text { E.P. } \\
\text { Notificados }\end{array}$} & \multicolumn{2}{|c|}{$\begin{array}{c}\text { E.P. } \\
\text { Calificados }\end{array}$} & \multirow[t]{2}{*}{ T.I. E.P } \\
\hline & $\mathrm{n}$ & $\%$ & $\mathrm{n}$ & $\%$ & & $\mathrm{n}$ & $\%$ & $\mathrm{n}$ & $\%$ & \\
\hline "Azuay & 797 & $3 \%$ & ב666 & $3 \%$ & 380,2 & 19 & $3 \%$ & 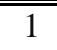 & $0 \%$ & 0,6 \\
\hline Bolívar & 131 & $1 \%$ & 126 & $1 \%$ & 532,1 & 3 & $0 \%$ & 0 & $0 \%$ & 0,0 \\
\hline Cañar & 528 & $2 \%$ & 336 & $2 \%$ & 813,5 & 4 & $1 \%$ & 0 & $0 \%$ & 0,0 \\
\hline Carchi & 54 & $0 \%$ & 54 & $0 \%$ & 215,6 & 0 & $0 \%$ & 0 & $0 \%$ & 0,0 \\
\hline
\end{tabular}

Revista Científica Mundo de la Investigación y el Conocimiento. 1(5). pp. 766-798 


\section{Responsabilidad social corporativa y su relación con la seguridad y salud ocupacional en el Ecuador}

Vol. 1, núm. 5., (2017)

Edison Daniel Yandun Burbano; Luis Fernando Freire Constante; Juan Carlos Flores; Carlos

Alberto Vasco Gualotuña

\begin{tabular}{lcccccccccc}
\hline Chimborazo & 173 & $1 \%$ & 137 & $1 \%$ & 178,9 & 2 & $0 \%$ & 0 & $0 \%$ & 0,0 \\
Cotopaxi & 356 & $2 \%$ & 326 & $2 \%$ & 388,3 & 38 & $5 \%$ & 0 & $0 \%$ & 0,0 \\
El Oro & 395 & $2 \%$ & 332 & $2 \%$ & 249,4 & 2 & $0 \%$ & 0 & $0 \%$ & 0,0 \\
Esmeraldas & 571 & $2 \%$ & 559 & $3 \%$ & 761,7 & 3 & $0 \%$ & 0 & $0 \%$ & 0,0 \\
Galápagos & 34 & $0 \%$ & 33 & $0 \%$ & 282,2 & 7 & $1 \%$ & 0 & $0 \%$ & 0,0 \\
Guayas & 9.911 & $43 \%$ & 9.823 & $47 \%$ & 1178,5 & 83 & $12 \%$ & 7 & $2 \%$ & 0,8 \\
Imbabura & 200 & $1 \%$ & 190 & $1 \%$ & 251,7 & 17 & $2 \%$ & 0 & $0 \%$ & 0,0 \\
Loja & 254 & $1 \%$ & 236 & $1 \%$ & 295,8 & 26 & $4 \%$ & 0 & $0 \%$ & 0,0 \\
Los Ríos & 1.022 & $4 \%$ & 259 & $1 \%$ & 236,8 & 5 & $1 \%$ & 0 & $0 \%$ & 0,0 \\
Manabí & 963 & $4 \%$ & 872 & $4 \%$ & 421,2 & 13 & $2 \%$ & 0 & $0 \%$ & 0,0 \\
Morona Santiago & 89 & $0 \%$ & 51 & $0 \%$ & 309,2 & 0 & $0 \%$ & 0 & $0 \%$ & 0,0 \\
Napo & 520 & $2 \%$ & 358 & $2 \%$ & 2740,4 & 6 & $1 \%$ & 0 & $0 \%$ & 0,0 \\
Orellana & 221 & $1 \%$ & 66 & $0 \%$ & 271,2 & 9 & $1 \%$ & 0 & $0 \%$ & 0,0 \\
Pastaza & 65 & $0 \%$ & 65 & $0 \%$ & 562,8 & 4 & $1 \%$ & 0 & $0 \%$ & 0,0 \\
Pichincha & 5.560 & $24 \%$ & 5.389 & $26 \%$ & 490,0 & 394 & $56 \%$ & 357 & $98 \%$ & 32,5 \\
Santa Elena & 200 & $1 \%$ & 198 & $1 \%$ & 822,4 & 5 & $1 \%$ & 0 & $0 \%$ & 0,0 \\
Santo Domingo & 333 & $1 \%$ & 328 & $2 \%$ & 627,1 & 19 & $3 \%$ & 0 & $0 \%$ & 0,0 \\
Tsáchilas & 23.093 & $100 \%$ & 20.983 & $100 \%$ & 630,7 & 704 & $100 \%$ & 365 & $100 \%$ & 11,0 \\
Sucumbíos & 258 & $1 \%$ & 212 & $1 \%$ & 921,1 & 21 & $3 \%$ & 0 & $0 \%$ & 0,0 \\
Tungurahua & 320 & $1 \%$ & 248 & $1 \%$ & 252,2 & 16 & $2 \%$ & 0 & $0 \%$ & 0,0 \\
Zamora Chinchipe & 138 & $1 \%$ & 119 & $1 \%$ & 808,1 & 8 & $1 \%$ & 0 & $0 \%$ & 0,0 \\
\hline & & & & & & & & $0 \%$ \\
\hline
\end{tabular}

Fuente: Datos obtenidos a través de la página web del Instituto Ecuatoriano de Seguridad Social (IESS) - Seguro General de Riesgos del Trabajo (SGRT) - Estadísticas del Seguro de Riesgos del Trabajo para el período 2013 a 2015. http://sart.iess.gob.ec/SRGP/indicadores_ecuador.php Consulta: 21 de abril de 2016.

Tabla 4 Tasa de incidencia (TI) de accidentes de trabajo (AT) y enfermedades profesionales (E.P.) según provincia, año 2015.

\begin{tabular}{|c|c|c|c|c|c|c|c|c|c|c|}
\hline \multirow{2}{*}{ Provincia } & \multicolumn{2}{|c|}{ A.T. } & \multicolumn{2}{|c|}{ A.T. } & $\begin{array}{l}\text { T.I. } \\
\text { A.T. }\end{array}$ & \multicolumn{2}{|c|}{$\begin{array}{c}\text { E.P. } \\
\text { Notificados }\end{array}$} & \multicolumn{2}{|c|}{$\begin{array}{c}\text { E.P. } \\
\text { Calificados }\end{array}$} & \multirow[t]{2}{*}{$\begin{array}{l}\text { T.I. } \\
\text { E.P. }\end{array}$} \\
\hline & $\mathrm{n}$ & $\%$ & $\mathrm{n}$ & $\%$ & & $\mathrm{n}$ & $\%$ & $\mathrm{n}$ & $\%$ & \\
\hline Azuay & 1.018 & $4 \%$ & 807 & $4 \%$ & 437,6 & 13 & $1 \%$ & 5 & $1 \%$ & 2,7 \\
\hline Bolívar & 111 & $0 \%$ & 102 & $0 \%$ & 626,7 & 3 & $0 \%$ & 0 & $0 \%$ & 0,0 \\
\hline Cañar & 667 & $3 \%$ & 507 & $2 \%$ & 1376,7 & 5 & $1 \%$ & 0 & $0 \%$ & 0,0 \\
\hline
\end{tabular}

Revista Científica Mundo de la Investigación y el Conocimiento. 1(5).pp. 766-798 


\section{Responsabilidad social corporativa y su relación con la seguridad y salud ocupacional en el Ecuador}

Vol. 1, núm. 5., (2017)

Edison Daniel Yandun Burbano; Luis Fernando Freire Constante; Juan Carlos Flores; Carlos Alberto Vasco Gualotuña

\begin{tabular}{|c|c|c|c|c|c|c|c|c|c|c|}
\hline Carchi & 60 & $0 \%$ & 54 & $0 \%$ & 290,5 & 2 & $0 \%$ & 0 & $0 \%$ & 0,0 \\
\hline Chimborazo & 236 & $1 \%$ & 231 & $1 \%$ & 436,6 & 19 & $2 \%$ & 0 & $0 \%$ & 0,0 \\
\hline Cotopaxi & 322 & $1 \%$ & 301 & $1 \%$ & 530,5 & 29 & $3 \%$ & 0 & $0 \%$ & 0,0 \\
\hline El Oro & 437 & $2 \%$ & 435 & $2 \%$ & 395,3 & 11 & $1 \%$ & 0 & $0 \%$ & 0,0 \\
\hline Esmeraldas & 549 & $2 \%$ & 523 & $2 \%$ & 1036,1 & 4 & $0 \%$ & 0 & $0 \%$ & 0,0 \\
\hline Galápagos & 47 & $0 \%$ & 32 & $0 \%$ & 369,6 & 0 & $0 \%$ & 0 & $0 \%$ & 0,0 \\
\hline Guayas & 10.321 & $42 \%$ & 9.819 & $44 \%$ & 1163,6 & 98 & $11 \%$ & 37 & $7 \%$ & 4,4 \\
\hline Imbabura & 149 & $1 \%$ & 143 & $1 \%$ & 231,8 & 12 & $1 \%$ & 0 & $0 \%$ & 0,0 \\
\hline Loja & 278 & $1 \%$ & 224 & $1 \%$ & 358,9 & 16 & $2 \%$ & 0 & $0 \%$ & 0,0 \\
\hline Los Ríos & 1.219 & $5 \%$ & 849 & $4 \%$ & 1136,6 & 5 & $1 \%$ & 0 & $0 \%$ & 0,0 \\
\hline Manabí & 860 & $4 \%$ & 817 & $4 \%$ & 365,9 & 9 & $1 \%$ & 0 & $0 \%$ & 0,0 \\
\hline Morona Santiago & 52 & $0 \%$ & 45 & $0 \%$ & 289,0 & 1 & $0 \%$ & 0 & $0 \%$ & 0,0 \\
\hline Napo & 506 & $2 \%$ & 502 & $2 \%$ & 3580,9 & 13 & $1 \%$ & 0 & $0 \%$ & 0,0 \\
\hline Orellana & 200 & $1 \%$ & 149 & $1 \%$ & 840,5 & 34 & $4 \%$ & 0 & $0 \%$ & 0,0 \\
\hline Pastaza & 111 & $0 \%$ & 105 & $0 \%$ & 846,4 & 10 & $1 \%$ & 0 & $0 \%$ & 0,0 \\
\hline Pichincha & 5.707 & $23 \%$ & 5.502 & $24 \%$ & 514,7 & 535 & $60 \%$ & 462 & $92 \%$ & 43,2 \\
\hline Santa Elena & 219 & $1 \%$ & 165 & $1 \%$ & 592,8 & 5 & $1 \%$ & 0 & $0 \%$ & 0,0 \\
\hline $\begin{array}{l}\text { Santo Domingo } \\
\text { Tsáchilas }\end{array}$ & 425 & $2 \%$ & 385 & $2 \%$ & 721,8 & 12 & $1 \%$ & 0 & $0 \%$ & 0,0 \\
\hline Sucumbíos & 264 & $1 \%$ & 260 & $1 \%$ & 1038,9 & 27 & $3 \%$ & 0 & $0 \%$ & 0,0 \\
\hline Tungurahua & 406 & $2 \%$ & 340 & $2 \%$ & 364,7 & 21 & $2 \%$ & 0 & $0 \%$ & 0,0 \\
\hline Zamora Chinchipe & 215 & $1 \%$ & 197 & $1 \%$ & 1188,8 & 8 & $1 \%$ & 0 & $0 \%$ & 0,0 \\
\hline & 24.379 & $100 \%$ & 22.494 & $100 \%$ & 715,1 & 892 & $100 \%$ & 504 & $100 \%$ & 16,0 \\
\hline
\end{tabular}

Fuente: Datos obtenidos a través de la página web del Instituto Ecuatoriano de Seguridad Social (IESS) - Seguro General de Riesgos del Trabajo (SGRT) - Estadísticas del Seguro de Riesgos del Trabajo para el período 2013 a 2015.

http://sart.iess.gob.ec/SRGP/indicadores_ecuador.php Consulta: 21 de abril de 2016

Analizando la información mostrada, tentativamente se verifica un incremento en cantidad de trabajadores afiliados, cantidad de accidentes reportados y la tasa de incidencia, a esto debemos aclarar que no solo es que se ha incrementado el índice, además se ha incrementado la cultura de reportar los accidentes laborales. De todas maneras, esta información 


\section{Responsabilidad social corporativa y su relación con la seguridad y salud ocupacional en el Ecuador}

Vol. 1, núm. 5., (2017)

Edison Daniel Yandun Burbano; Luis Fernando Freire Constante; Juan Carlos Flores; Carlos

Alberto Vasco Gualotuña

demuestra que la necesidad de una gestión técnica en los temas de salud y seguridad laboral es prioritaria para garantizar el buen vivir de la sociedad.

Gráfico 5 Cuadro comparativo y tendencia de los at y e.p., 2013 - 2015
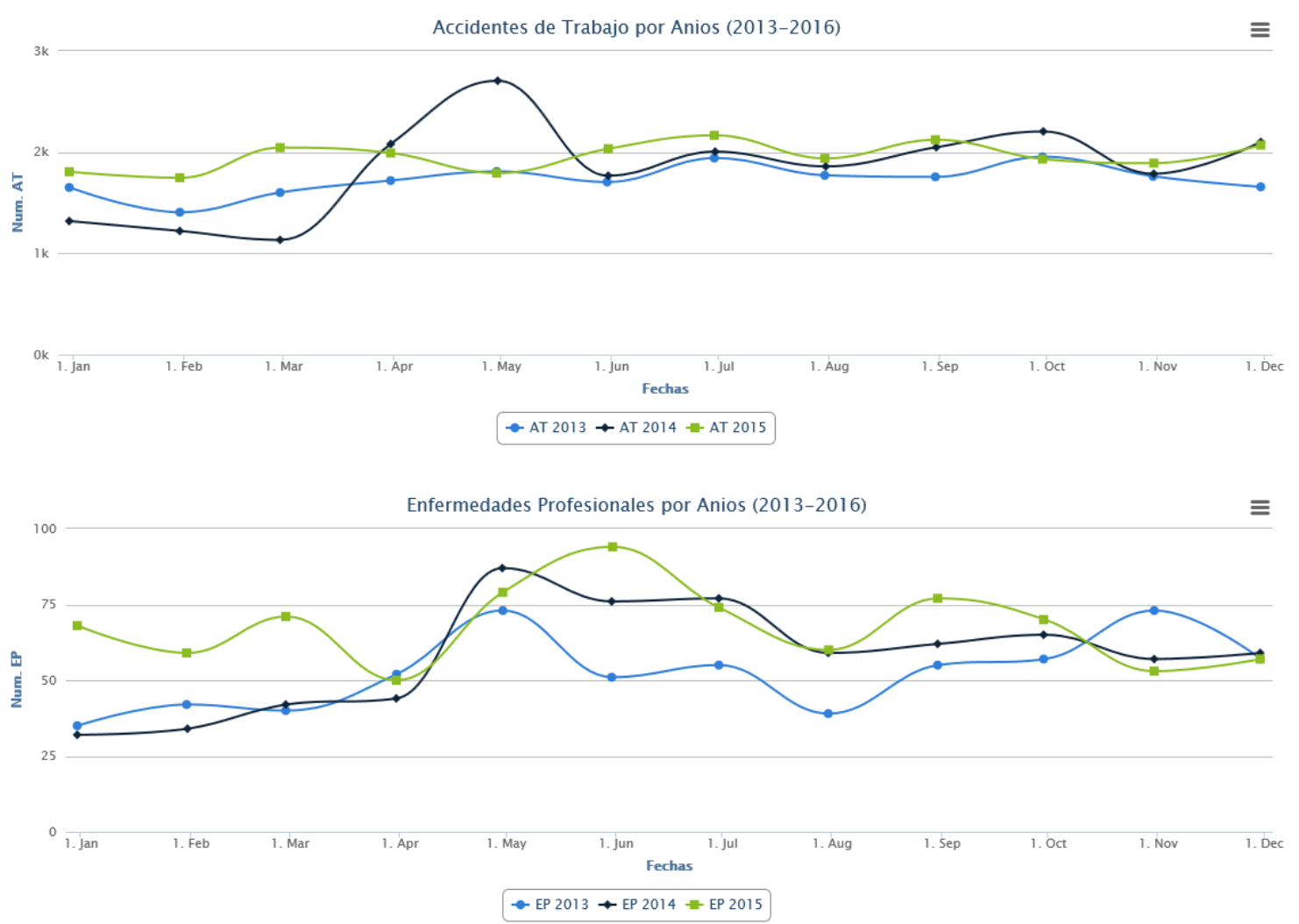

Fuente: Datos obtenidos a través de la página web del Instituto Ecuatoriano de Seguridad Social (IESS) - Seguro General de Riesgos del Trabajo (SGRT) - Estadísticas del Seguro de Riesgos del Trabajo para el período 2013 a 2015.

http://sart.iess.gob.ec/SRGP/indicadores_ecuador.php Consulta: marzo de 2017

Además, se pueden realizar análisis donde se determine las actividades económicas donde más se materialicen los riesgos por medio de accidentes y/o enfermedades laborales, y así 


\section{Responsabilidad social corporativa y su relación con la seguridad y salud ocupacional en el Ecuador}

Vol. 1, núm. 5., (2017)

Edison Daniel Yandun Burbano; Luis Fernando Freire Constante; Juan Carlos Flores; Carlos Alberto Vasco Gualotuña

priorizar la gestión hacia esas actividades. Esto sin tomar en cuenta que con el cambio de matriz productiva se desarrollaran nuevas actividades económicas que plantearan nuevos retos en la gestión de la seguridad y salud ocupacional.

Con estos antecedentes indicados desde la Carta fundamental del Estado Ecuatoriano, el Plan del Buen Vivir 2013 - $2017^{3}$ determina la estrategia de planificación del país, y por tanto los problemas y necesidades del mismo. El Plan del Buen Vivir 2013 - 2017 del estado Ecuatoriano establece unos objetivos y unas metas respecto del cuidado y protección del trabajador en el Ecuador estos objetivos son:

Objetivo 2; Auspiciar la igualdad, la cohesión, la inclusión y la equidad social y territorial, en la diversidad. El objetivo cuenta con unas políticas bien definidas, entre las cuales nos sentimos comprometidos a aportar desde nuestro campo específicamente en las siguientes:

2.6. Garantizar la protección especial universal y de calidad, durante el ciclo de vida, a personas en situación de vulneración de derechos.

b. Implementar mecanismos eficaces y permanentes de prevención, vigilancia y control del maltrato, explotación laboral, discriminación y toda forma de abuso y violencia contra niños, niñas y adolescentes.

Objetivo 3; Mejorar la calidad de vida de la población.

3 http://www.buenvivir.gob.ec/

Revista Científica Mundo de la Investigación y el Conocimiento. 1(5). pp. 766-798 


\section{Responsabilidad social corporativa y su relación con la seguridad y salud ocupacional en el Ecuador}

Vol. 1, núm. 5., (2017)

Edison Daniel Yandun Burbano; Luis Fernando Freire Constante; Juan Carlos Flores; Carlos

Alberto Vasco Gualotuña

3.2. Ampliar los servicios de prevención y promoción de la salud para mejorar las condiciones y los hábitos de vida de las personas. Fundamentalmente aportando en los siguientes lineamientos:

Diseñar e implementar mecanismos integrales de promoción de la salud para prevenir riesgos durante todo el ciclo de vida, con énfasis sobre los determinantes sociales de la salud.

Fortalecer el sistema de vigilancia y control epidemiológico, con corresponsabilidad comunitaria, ante posibles riesgos que causen morbilidad y mortalidad evitable o que sean de notificación obligatoria.

Implementar acciones integrales para la disminución de la morbilidad y la mortalidad por enfermedades transmisibles y crónicas no transmisibles o degenerativas de alta prioridad, y enfermedades evitables y desatendidas, por consumo de alimentos contaminados y por exposición a agroquímicos u otras sustancias tóxicas.

i. Promover la educación para la salud como principal estrategia para lograr el autocuidado y la modificación de conductas hacia hábitos de vida saludables.

n. Impulsar la creación de programas de medicina preventiva.

3.8. Propiciar condiciones adecuadas para el acceso a un hábitat seguro e incluyente.

Difundir y vigilar el cumplimiento de las normas y los estándares de comportamiento humano, seguridad, protección y producción de bienes o servicios, para prevenir y evitar posibles daños y/o contaminación ambiental, así como la propagación de epidemias o casos de violencia.

Revista Científica Mundo de la Investigación y el Conocimiento. 1(5). pp. 766-798 


\section{Responsabilidad social corporativa y su relación con la seguridad y salud ocupacional en el Ecuador}

Vol. 1, núm. 5., (2017)

Edison Daniel Yandun Burbano; Luis Fernando Freire Constante; Juan Carlos Flores; Carlos Alberto Vasco Gualotuña

3.11. Garantizar la preservación y protección integral del patrimonio cultural y natural y de la ciudadanía ante las amenazas y riesgos de origen natural o antrópico.

a. Diseñar e implementar normativas para prevenir, gestionar y mitigar los riesgos y desastres de origen natural o antrópico.

Incorporar la gestión integral, preventiva y sustentable de riesgos en los procesos de planificación y ordenamiento territorial nacional y local, para reducir la vulnerabilidad de las poblaciones ante las amenazas.

Coordinar y articular el sistema nacional descentralizado de gestión de riesgos, mejorando las capacidades institucionales y sociales, la producción de conocimiento y el intercambio de información científico-técnica.

Fortalecer la participación y las capacidades de respuesta ciudadana para fortalecer el Sistema Nacional Descentralizado de Gestión de Riesgos ante Desastres.

Mejorar los sistemas de control y alerta temprana, monitoreo y atención oportuna a la población, para identificar y mitigar las amenazas y vulnerabilidades sociales y ambientales ante los riesgos naturales y antrópicos.

Ampliar las capacidades del sector de seguridad, defensa y gestión de riesgos para la atención, rehabilitación y recuperación de las poblaciones, el patrimonio natural y las infraestructuras afectadas por desastres naturales o antrópicos.

Revista Científica Mundo de la Investigación y el Conocimiento. 1(5). pp. 766-798 


\section{Responsabilidad social corporativa y su relación con la seguridad y salud ocupacional en el Ecuador}

Vol. 1, núm. 5., (2017)

Edison Daniel Yandun Burbano; Luis Fernando Freire Constante; Juan Carlos Flores; Carlos

Alberto Vasco Gualotuña

Objetivo 4; Fortalecer las capacidades y potencialidades de la ciudadanía.

4.5. Potenciar el rol de docentes y otros profesionales de la educación como actores clave en la construcción del Buen Vivir.

Mejorar progresivamente la calidad de la educación, con un enfoque de derechos, de género, intercultural e inclusiva, para fortalecer la unidad en la diversidad e impulsar la permanencia en el sistema educativo y la culminación de los estudios.

Fortalecer la educación superior con visión científica y humanista, articulada a los objetivos para el Buen Vivir. Promover la investigación y el conocimiento científico, la revalorización de conocimientos y saberes ancestrales, y la innovación tecnológica.

4.6. Promover la interacción recíproca entre la educación, el sector productivo y la investigación científica y tecnológica, para la transformación de la matriz productiva y la satisfacción de necesidades.

Fortalecer y promocionar la formación técnica y tecnológica en áreas prioritarias y servicios esenciales para la transformación de la matriz productiva, considerando los beneficios del sistema dual de formación.

Objetivo 7; Garantizar los derechos de la naturaleza y promover la sostenibilidad ambiental, territorial y global. 


\section{Responsabilidad social corporativa y su relación con la seguridad y salud ocupacional en el Ecuador}

Vol. 1, núm. 5., (2017)

Edison Daniel Yandun Burbano; Luis Fernando Freire Constante; Juan Carlos Flores; Carlos Alberto Vasco Gualotuña

7.8. Prevenir, controlar y mitigar la contaminación ambiental en los procesos de extracción, producción, consumo y pos-consumo.

k. Fortalecer los mecanismos y las capacidades institucionales nacionales y locales para prevenir y controlar la contaminación de aire, suelo y agua, así como para garantizar la reparación integral de los danos y pasivos socio-ambientales que se generen.

Objetivo 8; Consolidar el sistema económico social y solidario, de forma sostenible

8.10. Articular la relación entre el estado y el sector privado.

j. Contratar trabajadores locales en los diferentes niveles de encadenamiento productivo territoriales bajo condiciones dignas.

Objetivo 9; Garantizar el trabajo digno en todas sus formas.

9.3. Profundizar el acceso a condiciones dignas para el trabajo, la reducción progresiva de la informalidad y garantizar el cumplimiento de los derechos laborales.

Fortalecer la normativa y los mecanismos de control para garantizar condiciones dignas en el trabajo, estabilidad laboral de los trabajadores y las trabajadoras, así como el estricto cumplimiento de los derechos laborales sin ningún tipo de discriminación.

e. Establecer mecanismos que aseguren entornos laborales accesibles y que ofrezcan condiciones saludables y seguras, que prevengan y minimicen los riesgos del trabajo.

Revista Científica Mundo de la Investigación y el Conocimiento. 1(5). pp. 766-798 


\section{Responsabilidad social corporativa y su relación con la seguridad y salud ocupacional en el Ecuador}

Vol. 1, núm. 5., (2017)

Edison Daniel Yandun Burbano; Luis Fernando Freire Constante; Juan Carlos Flores; Carlos

Alberto Vasco Gualotuña

Promover medidas que impulsen la existencia y el funcionamiento de organizaciones de trabajadoras y trabajadores, que permitan garantizar el cumplimiento de los derechos y obligaciones laborales.

Objetivo 10; Impulsar la transformación de la matriz productiva

10.3. Diversificar y generar mayor valor agregado en los sectores prioritarios que proveen servicios.

Fomentar la generación de capacidades técnicas y de gestión en los servicios, para mejorar su prestación y contribuir a la transformación productiva.

Objetivo 12; Garantizar la soberanía y la paz, inserción estratégica en el mundo y la integración latinoamericana.

El estado ecuatoriano por medio de este plan nacional busca entre otros cometidos mecanismos regionales alternativos y soberanos para la promoción y protección de los trabajadores y garantizar los Derechos Humanos, misión que compromete a las empresas en todo nivel.

\section{Conclusiones.}

El espíritu de la norma ISO 26000 en el Asunto 4 sobre prácticas laborales es promover y mantener el más alto grado de bienestar físico, mental y social de los trabajadores y prevenir los daños en la salud provocados por las condiciones laborales; ante esto, Tras la realización de la comparación respectiva se concluye que la legislación ecuatoriana mediante los cuerpos legales

Revista Científica Mundo de la Investigación y el Conocimiento. 1(5). pp. 766-798 


\section{Responsabilidad social corporativa y su relación con la seguridad y salud ocupacional en el Ecuador}

Vol. 1, núm. 5., (2017)

Edison Daniel Yandun Burbano; Luis Fernando Freire Constante; Juan Carlos Flores; Carlos Alberto Vasco Gualotuña

existentes cumple con lo manifestado por ISO 26000 que es de carácter voluntario. Es decir que todas las organizaciones ecuatorianas que cumplen con la parte legal en S.S.O. cumplen con los principios enmarcados en la norma ISO 26000.

Así en Ecuador es de cumplimiento obligatorio el gestionar la seguridad y salud laboral por parte de la alta dirección en donde es clave su compromiso; entre otras cuestiones, el formular la Política de Seguridad y Salud pues se marca un objetivo empresarial en esta materia garantizando recursos y, manifestando el compromiso de cumplir con la legislación técnico legal vigente en el país y el de garantizar las óptimas condiciones de trabajo.

La dirección empresarial es responsable de la creación y supervisión de la Unidad de Seguridad y Salud, esto aplica para empresas con 100 o más trabajadores o centros de trabajo calificados de alto riesgo cuando cuenten con más de 50 trabajadores. Esta Unidad de Seguridad y Salud que se forma desde un punto de vista preventivo, es dirigida por un profesional técnico en la materia, el cual se encarga de identificar, medir, evaluar y controlar los riesgos laborales sean estos en la fuente, medio y receptor y, crear programas que incluyan políticas preventivas. En la parte de Salud Ocupacional existe un servicio médico de empresa el cual se enfoca en prevenir posibles enfermedades laborales.

El instrumento legal de gestión lo desarrolla la Unidad de Seguridad y Salud con la aprobación de la Dirección y el Ministerio del Trabajo: es el Reglamento de Seguridad y Salud, en el cual prima el identificar y evaluar los riesgos existentes en la organización y plantea el 


\section{Responsabilidad social corporativa y su relación con la seguridad y salud ocupacional en el Ecuador}

Vol. 1, núm. 5., (2017)

Edison Daniel Yandun Burbano; Luis Fernando Freire Constante; Juan Carlos Flores; Carlos

Alberto Vasco Gualotuña

control de los mismos, crea responsabilidades preventivas a todo nivel así como los derechos y obligaciones de los miembros de una organización.

La participación de los trabajadores es fundamental en materia de seguridad y salud para lo cual las organizaciones que cuenten con más de quince trabajadores deben organizar un organismo paritario denominado Comité y/o Sub Comités de Seguridad y Salud conformado por representantes de los trabajadores y del empleador el cual se encarga entre otras funciones, de promover la observancia de las disposiciones sobre prevención de riesgos profesionales. Al finalizar un periodo se presenta a la Dirección y a los organismos de control como el Instituto Ecuatoriano de Seguridad Social o Ministerio de Trabajo los indicadores de gestión y actividades realizadas por los organismos paritarios.

Como continuación a la investigación, sería conveniente averiguar si estas acciones se están cumpliendo por parte de las organizaciones, así como si las Administraciones Públicas ecuatorianas implicadas realizan su debida vigilancia. En la misma línea, el estudio continuará con la detección de acciones/buenas prácticas activas de carácter voluntario realizadas por las empresas ecuatorianas no contempladas en la ISO 26000 (6.4.6.2), con el fin de hacerlas públicas para su conocimiento y puesta en marcha en otras organizaciones.

La Agencia Europea para la Seguridad y Salud en el trabajo propone como vía de mejora la PSLT (Promoción de la salud en el lugar de trabajo para los trabajadores), que comprende 4 fórmulas de avance en la calidad de vida del trabajador: 1: La mejora del método de organización del trabajo; 2: La mejora del entorno de trabajo; 3: Fomentar la participación de los empleados 


\section{Responsabilidad social corporativa y su relación con la seguridad y salud ocupacional en el Ecuador}

Vol. 1, núm. 5., (2017)

Edison Daniel Yandun Burbano; Luis Fernando Freire Constante; Juan Carlos Flores; Carlos Alberto Vasco Gualotuña

en actividades saludables; 4: Fomentar el desarrollo personal. Todas ellas implican el ir más allá del mero cumplimiento de los requisitos legales en materia de seguridad y salud en el trabajo correspondiéndose con una de las características fundamentales de la RS, su carácter voluntario y de evitación de impactos, de esta forma, las empresas contribuyen de manera activa la mejora de la salud y el bienestar general de sus trabajadores (Agencia Europea para la Seguridad y la Salud en el Trabajo, 2010).

\section{Bibliografía.}

Agencia Europea para la Seguridad y la Salud en el Trabajo. (2010). Promoción de la salud en el trabajo para los trabajadores. Recuperado el 2 de 12 de 2016, de http://osha.europa.eu/es/publications/factsheets/94

Comisión Europea. (2001). Libro Verde, Fomentar un marco europeo para la responsabilidad social de las empresas. Recuperado el 15 de noviembre de 2016, de http://eurlex.europa.eu/legal-content/ES/TXT/?uri=CELEX:52001DC0366

Comisión Europea. (2011). Estrategia renovada de la UE 2011-2014 sobre la responsabilidad social de las empresas. Bruselas.

Decreto Ejecutivo, D. E. (1986). El Reglamento de Seguridad y Salud de los Trabajadores y Mejoramiento del Ambiente de Trabajo. Quito.

ISO 26000. (2010). Recuperado el 25 de 11 de 2016, de https://www.iso.org/obp/ui\#iso:std:iso:26000:ed-1:v1:es

Nacional, A. (2008). CONSTITUCIÓN POLÍTICA DE LA REPÚBLICA DEL ECUADOR. Montecristi: Asamblea Nacional.

Organizacíón Internacional del Trabajo. (2006). Iniciativa InFocus sobre responsabilidad social de la empresa. Recuperado el 25 de 11 de 2016, de http://www.ilo.org/public/libdoc/ilo/GB/295/GB.295_MNE_2_1_span.pdf 